\title{
Effects of Engineered Saccharomyces cerevisiae Fermenting Cellobiose through Low-Energy-Consuming Phosphorolytic Pathway in Simultaneous Saccharification and Fermentation
}

\author{
Hyo-Jin Choi ${ }^{1}$, Yong-Su Jin ${ }^{2 *}$, and Won-Heong Lee ${ }^{1,2 *}$ \\ 'Department of Bioenergy Science and Technology, and Department of Integrative Food, Bioscience and \\ Biotechnology, Chonnam National University, Gwangju 61186, Republic of Korea \\ ${ }^{2}$ Department of Food Science and Human Nutrition, and Carl R. Woese Institute for Genomic Biology, University of \\ Illinois at Urbana-Champaign, Urbana, IL 61801, USA
}

Until recently, four types of cellobiose-fermenting Saccharomyces cerevisiae strains have been developed by introduction of a cellobiose metabolic pathway based on either intracellular $\beta$ glucosidase (GH1-1) or cellobiose phosphorylase (CBP), along with either an energy-consuming active cellodextrin transporter (CDT-1) or a non-energy-consuming passive cellodextrin facilitator (CDT-2). In this study, the ethanol production performance of two cellobiose-fermenting $S$. cerevisiae strains expressing mutant CDT-2 (N306I) with GH1-1 or CBP were compared with two cellobiose-fermenting S. cerevisiae strains expressing mutant CDT-1 (F213L) with GH1-1 or CBP in the simultaneous saccharification and fermentation (SSF) of cellulose under various conditions. It was found that, regardless of the SSF conditions, the phosphorolytic cellobiose-fermenting $S$. cerevisiae expressing mutant CDT-2 with CBP showed the best ethanol production among the four strains. In addition, during SSF contaminated by lactic acid bacteria, the phosphorolytic cellobiosefermenting S. cerevisiae expressing mutant CDT-2 with CBP showed the highest ethanol production and the lowest lactate formation compared with those of other strains, such as the hydrolytic cellobiose-fermenting S. cerevisiae expressing mutant CDT-1 with GH1-1, and the glucosefermenting $S$. cerevisiae with extracellular $\beta$-glucosidase. These results suggest that the cellobiosefermenting yeast strain exhibiting low energy consumption can enhance the efficiency of the SSF of cellulosic biomass.

Keywords: Cellulosic ethanol, simultaneous saccharification and fermentation, mutant cellodextrin facilitator, cellobiose phosphorylase, engineered Saccharomyces cerevisiae

\section{Introduction}

To produce cellulosic biofuels efficiently, the development of Saccharomyces cerevisiae-a useful host for bioethanol production-to utilize intracellular cellobiose has been proposed as a strategy for avoiding glucose repression that frequently reduces product yield and productivity during the fermentation of mixed sugars derived from cellulosic biomass [1-5]. Indeed, engineered S. cerevisiae, which is capable of fermenting cellobiose, exhibits enhanced ethanol yield and productivity through simultaneous fermentation of cellobiose and other sugars compared with ethanol yield and productivity observed through sequential fermentation of glucose and other sugars $[3,6,7]$. In particular, S. cerevisiae strains fermenting cellobiose require lower amounts of cellulolytic enzymes and exhibit similar or even better ethanol production performance than those of the non-engineered strain (the parental S. cerevisiae fermenting glucose degraded from cellobiose by supplementation of extracellular $\beta$-glucosidase) during simultaneous saccharification and fermentation (SSF) of cellulose [8-10].

For S. cerevisiae to ferment cellobiose, it is essential to introduce genes encoding cellodextrin transporters (CDTs) and intracellular cellobiose degrading enzymes-components of the heterologous cellobiose metabolic pathway [11-13]. Previously, two types of cellodextrin transporters were identified from Neurospora crassa: energy-dependent CDT-1, an active transporter spending one mole of ATP for transporting one mole of cellobiose [11, 14]; and energy-independent CDT-2, a facilitator that does not spend ATP for cellobiose transport $[11,14]$. Two types of intracellular cellobiose degrading enzymes have also been identified: intracellular $\beta$ - 
glucosidase (GH1-1) from Neurospora Crassa, involved in the cellobiose hydrolysis requiring two moles of ATP to initiate glycolysis [11, 14]; and cellobiose phosphorylase (CBP) from Saccharophagus degradans, involved in the cellobiose phosphorolysis requiring only one mole of ATP to initiate glycolysis [12,15]. On this basis, four types of the cellobiose-fermenting S. cerevisiae strains have been developed, as shown in Fig. S1. (1) hydrolytic cellobiosefermenting S. cerevisiae with CDT-1 and GH1-1 (D-BT1 strain), (2) phosphorolytic cellobiose-fermenting $S$. cerevisiae with CDT-1 and CBP (D-CT1 strain), (3) hydrolytic cellobiose-fermenting S. cerevisiae with CDT-2 and GH1-1 (D-BT2 strain), and (4) phosphorolytic cellobiose-fermenting S. cerevisiae with CDT-2 and CBP (D-CT2 strain) $[11,12,14,15]$.

Although CDT-1 and CDT-2 exhibit similar affinity to cellobiose, CDT-1 demonstrates a two-fold higher cellobiose transporting activity than that of CDT- $2\left(\mathrm{~V}_{\max }\right.$ of CDT- $1 \approx 0.7 \mathrm{pmol} / \mathrm{s} \mathrm{vs} . \mathrm{V}_{\max }$ of CDT- $2 \approx 0.35 \mathrm{pmol} / \mathrm{s}$ ) [11]. The CBP-catalyzed reaction is thermodynamically unfavorable compared with the GH1-1-catalyzed reaction $\left(\Delta \mathrm{G}^{0}\right.$ of cellobiose phosphorolysis $=3.6 \mathrm{~kJ} / \mathrm{mol}$ vs. $\Delta \mathrm{G}^{0}$ of cellobiose hydrolysis $\left.=-12.5 \mathrm{~kJ} / \mathrm{mol}\right)[12,16$, 17]. In addition, although CBP and GH1-1 exhibit almost the same $V_{\max }$, CBP demonstrates a two-fold lower affinity to cellobiose than that of GH1-1 $\left(\mathrm{K}_{\mathrm{m}}\right.$ of CBP $=0.65 \mathrm{mM}$ vs. $\mathrm{K}_{\mathrm{m}}$ of GH1-1 $\left.=0.32 \mathrm{mM}\right)$ [12]. In summary, the D-BT1 strain has been considered as the best cellobiose-fermenting yeast strain $[12,14,15,18]$. Consequently, the priority of the cellobiose fermentation efficiencies of the cellobiose-fermenting yeast strains could be presented in the order of D-BT $1>\mathrm{D}$-CT $1 \approx \mathrm{D}-\mathrm{BT} 2>\mathrm{D}$-CT2 .

However, the cellobiose fermentation performance of the phosphorolytic cellobiose-fermenting S. cerevisiae strains can be significantly improved by employing mutant CDTs, such as CDT-1 (F213L) and CDT-2 (N306I), which exhibit three-fold and six-fold enhanced cellobiose transporting activities than those of the wild-type CDT1 and CDT-2, respectively $[12,15]$. The phosphorolytic cellobiose-fermenting $S$. cerevisiae strains expressing either mutant CDT-1 or mutant CDT-2 (D-CT1m and D-CT2m strains) exhibited a similar fermentation performance compared with that of the hydrolytic cellobiose-fermenting S. cerevisiae expressing mutant CDT-1 (D-BT1m strain) and an even better fermentation performance than that of the hydrolytic cellobiose-fermenting S. cerevisiae expressing mutant CDT-2 (D-BT2m strain) in the cellobiose fermentation under micro-aerobic conditions $[12,15]$. Consequently, the cellobiose fermentation efficiencies of the cellobiose-fermenting yeast strains with mutant transporters could be presented in the following order: D-BT1m $\approx D-C T 1 m \approx D-C T 2 m>D$ BT2m.

In the previous studies, in addition to cellobiose fermentation performance comparison, the ethanol production performance of the D-BT1m strain have been evaluated in the SSF of cellulose [8,9]. Although both D-BT1m and D-CT1m strains showed superior ethanol production than that of the non-engineered S. cerevisiae strain fermenting glucose by extracellular supplementation of $\beta$-glucosidase $[8,9]$, the D-CT1m strain exhibited a slightly lower ethanol production compared with that of the D-BT1m strain, indicating that the energetic advantage of CBP was barely observed in the case of cellobiose-fermenting yeast strain expressing mutant CDT-1 during the SSF of cellulose [9].

This study aimed to evaluate whether the D-BT2m strain, expected to demonstrate an energy-saving effect compared with that of mutant CDT-1, could perform similar or better ethanol production in the SSF of cellulose compared with that of the D-BT1m strain. To this end, the ethanol production performance of D-BT2m and D$\mathrm{CT} 2 \mathrm{~m}$ strains during SSF under various conditions were compared with that of D-BT1m and D-CT1m strains. In addition, the ethanol production of D-CT2m was compared with those of D-BT1m and the glucose-fermenting S. cerevisiae with extracellular $\beta$-glucosidase (D-56+188 strain) during SSF contaminated by lactic acid bacteria (LAB).

\section{Materials and Methods}

\section{Strains, Plasmids, and Culture Conditions}

S. cerevisiae D452-2 (Mata, leu2, his3, ura3 and can1) [19] was used as the host strain for overexpression of the GH1-1 from N. crassa or the CBP from Saccharophagus degradans along with either the mutant active CDT-1 (F213L) or the mutant passive cellodextrin facilitator [CDT-2 (N306I)] from N. crassa. Plasmids pRS425-gh1-1, pRS425-CBP, pRS426-cdt1 (F213L), and pRS426-cdt2 (N306I) were previously constructed for overexpression of GH1-1, CBP, CDT-1 (F213L), and CDT-2 (N306I), respectively [11, 12, 15]. All S. cerevisiae strains and the plasmids used in this study are listed in Table 1.

Synthetic complete (SC) medium (6.7 g/l of yeast nitrogen base without amino acids, $0.625 \mathrm{~g} / \mathrm{l}$ of complete supplement mixture without leucine and uracil, $\mathrm{pH}$ 6.0) containing $20 \mathrm{~g} / \mathrm{l}$ of glucose, SCD20, was used for seed cultivation. Yeast extract-peptone (YP) medium (10 g/l of yeast extract and $20 \mathrm{~g} / \mathrm{l}$ of Bacto-peptone, $\mathrm{pH}$ 6.7) containing $50 \mathrm{~g} / \mathrm{l}$ of cellobiose, YPC50, was used for pre-cultivation of the cellobiose-fermenting strains (DBT1m, D-CT1m, D-BT2m, and D-CT2m). YP medium containing $50 \mathrm{~g} / \mathrm{l}$ of glucose, YPD50, was used for precultivation of the glucose-fermenting strain expressing neither cellodextrin transporter nor cellobiose degrading enzyme (the parental strain containing empty plasmids, D-56 strain). Yeast cells at the exponential growth phase during the pre-cultivation were harvested and used for SSF. Seed cultivation and pre-cultivation were performed at $30^{\circ} \mathrm{C}$ and $250 \mathrm{rpm}$.

Lactobacillus fermentum KCTC 3112 was used as the contaminant during SSF with contamination by LAB. De Man, Rogosa and Sharpe (MRS) medium (10 g/l of peptone, $8 \mathrm{~g} / \mathrm{l}$ of meat extract, $4 \mathrm{~g} / \mathrm{l}$ of yeast extract, $2 \mathrm{~g} / \mathrm{l}$ of dipotassium hydrogen phosphate, $1 \mathrm{~g} / \mathrm{l}$ of polysorbate $80,1 \mathrm{~g} / \mathrm{l}$ of di-ammonium hydrogen citrate, $5 \mathrm{~g} / \mathrm{l}$ of sodium acetate, $0.2 \mathrm{~g} / \mathrm{l}$ of magnesium sulfate, $0.04 \mathrm{~g} / \mathrm{l}$ of manganese sulfate and $20 \mathrm{~g} / \mathrm{l}$ of glucose, $\mathrm{pH}$ 6.5) was used for seed cultivation and pre-cultivation of $L$. fermentum. LAB cells at the exponential growth phase in the pre-cultivation 
Table 1. Plasmids and S. cerevisiae strains used in this study.

\begin{tabular}{|c|c|c|}
\hline Plasmids and strains & Relevant features & Reference \\
\hline \multicolumn{3}{|l|}{ Plasmids } \\
\hline pRS425PGK & LEU2, $\mathrm{P}_{\mathrm{PGK}}-\mathrm{MCS}-\mathrm{T}_{\mathrm{CYC}}, 2 \mu$ origin, $\mathrm{Amp}^{\mathrm{r}}$ & {$[11]$} \\
\hline pRS425-gh1-1 & LEU2, $\mathrm{P}_{\mathrm{PGK}^{-}}-g h 1-1-\mathrm{T}_{\mathrm{CYC}}, 2 \mu$ origin, $\mathrm{Amp}^{\mathrm{r}}$ & [11] \\
\hline pRS425-CBP & LEU2, $\mathrm{P}_{\mathrm{PGK}^{-}} C B P-\mathrm{T}_{\mathrm{CYC}}, 2 \mu$ origin, $\mathrm{Amp}^{\mathrm{r}}$ & {$[12]$} \\
\hline pRS426PGK & $U R A 3, \mathrm{P}_{\mathrm{PGK}}-\mathrm{MCS}-\mathrm{T}_{\mathrm{CYC}}, 2 \mu$ origin, $\mathrm{Amp}^{\mathrm{r}}$ & {$[11]$} \\
\hline pRS426-cdt1 (F213L) & $U R A 3, \mathrm{P}_{\mathrm{PGK}}-c d t 1(\mathrm{~F} 213 \mathrm{~L})-\mathrm{T}_{\mathrm{CYC}}, 2 \mu$ origin, $\mathrm{Amp}^{\mathrm{r}}$ & {$[12]$} \\
\hline pRS426-cdt2 (N306I) & $U R A 3, \mathrm{P}_{\mathrm{PGK}}-c d t 2(\mathrm{~N} 306 \mathrm{I})-\mathrm{T}_{\mathrm{CYC}}, 2 \mu$ origin, $\mathrm{Amp}^{\mathrm{r}}$ & {$[15]$} \\
\hline \multicolumn{3}{|l|}{ Strains } \\
\hline D452-2 & MAT $\alpha$, leu2, his 3, ura 3 and can 1 & [19] \\
\hline D-56 & D452-2/pRS425PGK/pRS426PGK & {$[8]$} \\
\hline D- $56+188$ & D-56 with extracellular $\beta$-glucosidase & {$[8]$} \\
\hline D-BT1m & D452-2/pRS425-gh1-1/pRS426-cdt1 (F213L) & {$[12]$} \\
\hline D-CT1m & D452-2/pRS425-CBP/pRS426-cdt1 (F213L) & {$[12]$} \\
\hline $\mathrm{D}-\mathrm{BT} 2 \mathrm{~m}$ & D452-2/pRS425-gh1-1/pRS426-cdt2 (N306I) & [15] \\
\hline D-CT2m & D452-2/pRS425-CBP/pRS426-cdt2 (N306I) & {$[15]$} \\
\hline
\end{tabular}

were harvested and used for SSF with LAB contamination. Seed cultivation and pre-cultivation were performed at $30^{\circ} \mathrm{C}$ without agitation.

\section{Conditions for SSF of Cellulose}

Avicel PH-101 (Sigma, USA) was used as the substrate for SSF. Celluclast 1.5L (Novozyme, Denmark) was used as the cellulase mixture for the saccharification of cellulose during SSF. Novozyme 188 (Novozyme) was used as the extracellular $\beta$-glucosidase for degradation of cellobiose to glucose during SSF with the parental strain containing empty plasmids. The inoculum size of yeast cells was adjusted to an optical density of 30, measured at $600 \mathrm{~nm}(\mathrm{OD} 600)$ and corresponding to $10.5 \mathrm{~g} / \mathrm{l}$ of dry cell concentration, according to the condition determined in the previous studies [8-10].

SSF was carried out under three different conditions: (1) Micro-aerobic SSF was performed in 250-ml flasks filled with $50 \mathrm{ml}$ of YP medium containing Avicel PH-101 (130 g/l) and Celluclast 1.5L [10 filter paper unit (FPU)/ g cellulose], according to the conditions determined in the previous studies $[8,9]$. The flasks were equipped with an airlock device (three-piece airlock with silicone stopper), releasing $\mathrm{CO}_{2}$ with minimized air-inflow. The temperature and agitation speed were maintained at $30^{\circ} \mathrm{C}$ and $100 \mathrm{rpm}$, respectively; (2) Anaerobic SSF was performed in 125-ml serum bottles filled with $25 \mathrm{ml}$ of YP medium containing Avicel PH-101 (130 g/l) and Celluclast 1.5L (10 FPU/g cellulose). After purging the argon gas for $15 \mathrm{~min}$, the serum bottles were tightly sealed with rubber caps, completely blocking the air-inflow. The bottles inoculated with yeast cells were maintained at $30^{\circ} \mathrm{C}$ and $100 \mathrm{rpm}$, respectively; (3) Anaerobic SSF contaminated by LAB was performed under the same conditions as anaerobic SSF, except for the co-inoculation of yeast and LAB cells. Novozyme 188 [5.4 cellobiase unit (CBU)/g cellulose] was used as the extracellular $\beta$-glucosidase for degradation of cellobiose to glucose during SSF with the parental yeast strain fermenting only glucose. The inoculum size of LAB cells for contamination was adjusted to OD600 of 3, which is equivalent to OD600 of 30 for yeast cells [20]. All SSF experiments were performed in triplicate.

\section{Analytical Methods}

The concentrations of ethanol and lactate were determined by high-performance liquid chromatography (HPLC; Agilent Technologies 1200 Series, Agilent, USA) equipped with a refractive index (RI) detector using the Rezex ROA-Organic Acid H+ (8\%) column (Phenomenex Inc., USA). The column was eluted with $0.005 \mathrm{~N}$ of $\mathrm{H}_{2} \mathrm{SO}_{4}$ at a flow rate of $0.6 \mathrm{ml} / \mathrm{min}$ at $50^{\circ} \mathrm{C}$.

\section{Results}

Performance of the Cellobiose-Fermenting S. cerevisiae Strains Expressing CDT-1 or CDT-2 during MicroAerobic SSF

In the previous studies, the D-CT1m strain exhibited as good ethanol production as that of the D-BT1m strain in the cellobiose fermentation and the SSF of cellulose $[9,12]$. In another study, mutant CDT-2 was developed from wild-type CDT-2, whereby the D-CT2m strain fermented cellobiose as fast as the D-CT1m strain in the cellobiose fermentation [15]. These results suggested that the cellobiose-fermenting yeast strains expressing mutant CDT-2 (especially, D-CT2m) might demonstrate equivalent SSF performance to the cellobiosefermenting yeast strains expressing mutant CDT-1 (both D-BT1m and D-CT1m). Therefore, to verify the ethanol production performance of the cellobiose-fermenting $S$. cerevisiae strains expressing mutant CDT-2 (D-BT2m and D-CT2m), SSF experiments with four cellobiose-fermenting yeast strains expressing either mutant CDT-1 or mutant CDT-2 were performed.

Fig. 1 shows ethanol production profiles observed during micro-aerobic SSF of pure cellulose (Avicel PH-101) by four cellobiose-fermenting $S$. cerevisiae strains with mutant cellodextrin transporters.

When comparing ethanol production between two cellobiose-fermenting yeast strains expressing mutant CDT-2, D-CT2m showed considerably faster ethanol production than that of D-BT2m during the entire period of 


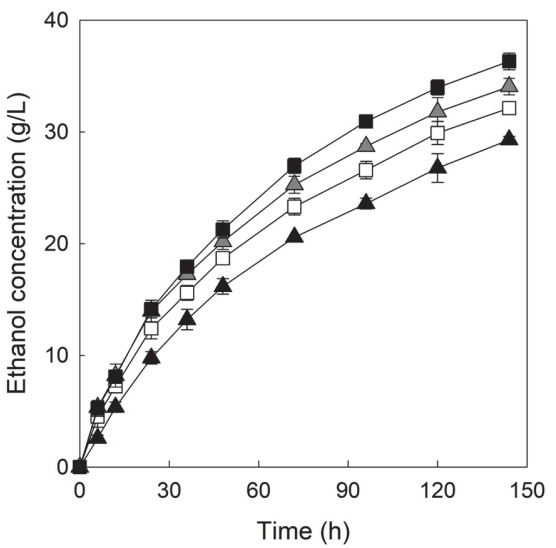

Fig. 1. Ethanol production profiles during micro-aerobic SSF of 13\% Avicel PH-101. SSF was performed by yeast cells with an OD600 value of 30 at $30^{\circ} \mathrm{C}$ and $100 \mathrm{rpm}$. Celluclast $1.5 \mathrm{~L}$ (10 FPU/g cellulose) was used for the saccharification of cellulose. The yeast strains used for the SSF are as follows: D-BT1m (the hydrolytic S. cerevisiae expressing mutant CDT-1 and GH1-1; grey triangle, $\mathbf{A}$ ); D-CT1m (the phosphorolytic S. cerevisiae expressing mutant CDT-1 and CBP; white square, $\square$ ); DBT2m (the hydrolytic S. cerevisiae expressing mutant CDT-2 and GH1-1; black triangle, $\mathbf{\Delta}$ ); D-CT2m (the phosphorolytic $S$. cerevisiae expressing mutant CDT-2 and CBP; black square, $\mathbf{\square})$. Ethanol concentration was measured in three independent experiments, and the symbols in the figure indicate average values with standard deviations.

SSF. The difference in ethanol production between the two strains increased over time. Consequently, D-CT2m produced $24 \%$ more final ethanol than that produced by D-BT2m $(36.3 \mathrm{~g} / \mathrm{l}$ of ethanol by D-CT2m vs. $29.3 \mathrm{~g} / \mathrm{l}$ of ethanol by D-BT2m), as shown in Table 2. Even though D-BT2m and D-CT2m expressed the same transporter, mutant CDT-2, the ethanol production by D-BT2m was considerably lower than that of D-CT2m. This is consistent with the previous study, which demonstrated a considerably lower ethanol yield and productivity by D$\mathrm{BT} 2 \mathrm{~m}$ compared with those achieved by $\mathrm{D}-\mathrm{CT} 2 \mathrm{~m}$ during the micro-aerobic cellobiose fermentation [15]. As yeast cells might generate sufficient energy in forms such as ATP by respiration under micro-aerobic conditions, the energetic benfit of the CBP-catalyzed reaction (saving one mole of ATP for degrading one mole of cellobiose compared with GH1-1-catalyzed reaction) might have little effect during SSF under micro-aerobic conditions $[12,15]$. The enhancement of the ethanol production by D-CT2 $\mathrm{m}$ over that of D-BT2 $\mathrm{m}$ might be due to the $33 \%$ higher maximum specific growth rate $\left(\mu_{\max }\right)$ of D-CT2m for cellobiose than that of D-BT2m under micro-aerobic conditions, as shown in Fig. S2.

Meanwhile, when comparing ethanol production of two cellobiose-fermenting yeast strains expressing mutant CDT-1, D-BT $1 \mathrm{~m}$ produced $6 \%$ more final ethanol than that produced by D-CT1m during SSF $(34.0 \mathrm{~g} / \mathrm{l}$ of ethanol by D-BT1m vs.32.1 g/l of ethanol by D-CT1m), which is a consistent pattern with the results from the previous study [9].

Interestingly, when comparing the ethanol production by D-CT2m with those achieved by cellobiosefermenting strains expressing mutant CDT-1, D-CT2m showed faster ethanol production than those of both DBT1m and D-CT1m. Although D-CT2m produced a similar amount of ethanol compared with those of other strains (D-BT1m and D-CT1m) up to $12 \mathrm{~h}$ of SSF, subsequently, it produced more ethanol than that produced by other strains. The final concentration of ethanol produced by D-CT2m was $7 \%$ and $13 \%$ higher than those

Table 2. Summary of the results from the SSF of cellulose performed by S. cerevisiae strains fermenting cellobiose.

\begin{tabular}{lcccc}
\hline \multicolumn{1}{c}{ Culture conditions } & Strains & $\begin{array}{c}\text { Final ethanol } \\
(\mathrm{g} / \mathrm{l})\end{array}$ & $\begin{array}{c}\text { Final lactate } \\
(\mathrm{g} / \mathrm{l})\end{array}$ & $\begin{array}{c}\text { Ethanol yield from cellulose } \\
(\mathrm{g} / \mathrm{g})\end{array}$ \\
\hline Micro-aerobic, 13\% Avicel, & D-BT1m & $34.0 \pm 0.74$ & - & 0.29 \\
Celluclast 1.5L (10 FPU/g cellulose) & D-CT1m & $32.1 \pm 0.56$ & - & 0.27 \\
& D-BT2m & $29.3 \pm 0.31$ & - & 0.25 \\
& D-CT2m & $36.3 \pm 0.75$ & - & 0.31 \\
Anaerobic, 13\% Avicel, & D-BT1m & $35.1 \pm 0.38$ & - & 0.30 \\
Celluclast 1.5L (10 FPU/g cellulose) & D-CT1m & $34.1 \pm 0.29$ & - & 0.29 \\
& D-BT2m & $27.6 \pm 1.17$ & - & 0.23 \\
Anaerobic, 13\% Avicel, & D-CT2m & $36.3 \pm 0.40$ & - & 0.31 \\
Celluclast 1.5L (10 FPU/g cellulose), & D-56+188 & $19.9 \pm 0.08$ & $10.1 \pm 0.13$ & 0.17 \\
L.fermentum ((OD600 3)) & D-BT1m & $19.0 \pm 0.03$ & $9.4 \pm 0.09$ & 0.16 \\
\hline
\end{tabular}

${ }^{*}$ Since Avicel exhibits 9\% water content, as shown in the previous study [8], the ethanol yield from $13 \%$ Avicel was calculated based on the final ethanol concentration divided by $118.3 \mathrm{~g} / \mathrm{l}$ of actual Avicel concentration. In SSF with D-56+188, Novozyme 188 (5.4 CBU/g cellulose) was added along with Celluclast 1.5L for degradation of cellobiose to glucose. 
produced by D-BT1m and D-CT1m, respectively (Table 2). Similar to the CBP-catalyzed reaction, the energetic benefit of cellobiose transport based on the mutant CDT-2 (saving one mole of ATP for transporting one mole of cellobiose compared with the mutant CDT-1) might show little effect under the micro-aerobic condition $[12,15]$. Therefore, the enhanced ethanol production by D-CT2 $\mathrm{m}$ than those of both D-BT1m and D-CT1m might be attributed to the observation that D-CT2m showed a faster specific growth rate for cellobiose than those of other yeast strains expressing mutant CDT-1 under micro-aerobic conditions (Fig. S2). The final concentrations and yields of ethanol produced by cellobiose-fermenting S. cerevisiae expressing mutant cellodextrin transporters are summarized in Table 2.

\section{Performance of the Cellobiose-Fermenting S. cerevisiae Strains Expressing CDT-1 or CDT-2 during Anaerobic SSF}

Compared with micro-aerobic SSF, anaerobic SSF was also performed to confirm whether there was a change in ethanol production by cellobiose-fermenting S. cerevisiae strains expressing mutant cellodextrin transporters when the oxygen supply was tightly restricted.

Fig. 2 shows ethanol production profiles observed during anaerobic SSF of Avicel PH-101 by four cellobiosefermenting S. cerevisiae strains with either mutant CDT-1 or mutant CDT-2.

When comparing ethanol production between two cellobiose-fermenting yeast strains expressing mutant CDT-2, D-CT2m showed a faster and higher ethanol production than that of D-BT2m throughout SSF, similar to that observed during micro-aerobic SSF. The gap of ethanol production between D-CT2 $\mathrm{m}$ and D-BT2m expanded over time from an initial value of $3.3 \mathrm{~g} / \mathrm{l}$ (at $12 \mathrm{~h}$ of SSF) to a final value of $8.7 \mathrm{~g} / \mathrm{l}$ (at $144 \mathrm{~h}$ of SSF). As shown in Table 2, D-CT2m produced $36.3 \mathrm{~g} / \mathrm{l}$ of ethanol, which was $31 \%$ higher than the amount of ethanol produced by D-BT2m. Notably, the difference in ethanol production between D-CT2m and D-BT2m during anaerobic SSF was larger than that observed during micro-aerobic SSF. These results suggest that the energetic benefit of D-CT2 $\mathrm{m}$ based on CBP might become potent during anaerobic SSF involving limited energy generation by the yeast cells $[12,15]$. Hence, compared with micro-aerobic SSF, D-CT2m seemed to produce a similar amount of ethanol during anaerobic SSF, whereas D-BT2m produced less amount of ethanol under SSF conditions with restricted aeration.

When comparing ethanol production between two cellobiose-fermenting strains expressing mutant CDT-1, DBT1m showed a higher ethanol production than that of D-CT1m $(35.1 \mathrm{~g} / 1$ of ethanol by D-BT1m vs.34.1 g/l of ethanol by D-CT1m), similar to that observed during micro-aerobic SSF, consistent with the results from the previous studies $[8,9]$.

Similar to the results from micro-aerobic SSF, D-CT2m showed faster and higher ethanol production than those achieved by cellobiose-fermenting strains expressing mutant CDT-1. D-CT2m produced a similar level of ethanol compared with those of D-BT1m and D-CT1m during the early period of SSF (until $24 \mathrm{~h}$ of SSF). However, D-CT2 $\mathrm{m}$ produced more ethanol than those produced by D-BT1m and D-CT1m from the middle of SSF (after $72 \mathrm{~h}$ of SSF). The final concentration of ethanol produced by D-CT $2 \mathrm{~m}$ was $3 \%$ and $6 \%$ higher than those produced by D-BT1 $\mathrm{m}$ and D-CT1 $\mathrm{m}$, respectively (Table 2). As mentioned above, enhanced ethanol production by $\mathrm{D}-\mathrm{CT} 2 \mathrm{~m}$ than those by other strains expressing mutant CDT-1 might be attributed to the energy-saving effects based on both mutant CDT-2 and CBP $[12,15]$. When considering that the gap of ethanol production between D$\mathrm{CT} 2 \mathrm{~m}$ and $\mathrm{D}-\mathrm{CT} 1 \mathrm{~m}$ was higher than that between $\mathrm{D}-\mathrm{CT} 2 \mathrm{~m}$ and $\mathrm{D}-\mathrm{BT} 1 \mathrm{~m}$, it might be reasonable to state that the

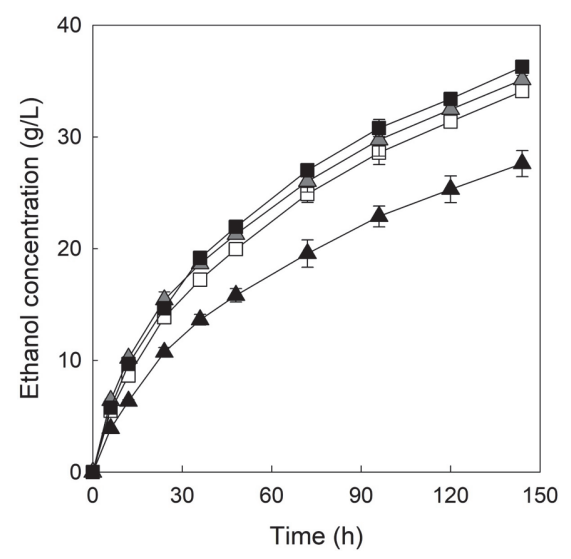

Fig. 2. Ethanol production profiles during anaerobic SSF of $13 \%$ Avicel PH-101. SSF was performed by yeast cells with an OD600 value of 30 at $30^{\circ} \mathrm{C}$ and $100 \mathrm{rpm}$. Celluclast $1.5 \mathrm{~L}$ (10 FPU/g cellulose) was used for the saccharification of cellulose. The yeast strains used for the SSF are as follows: D-BT1m (the hydrolytic S. cerevisiae expressing mutant CDT-1 and GH1-1; grey triangle, $\mathbf{\Delta}$ ); D-CT1m (the phosphorolytic S. cerevisiae expressing mutant CDT-1 and CBP; white square, $\square$ ); DBT2m (the hydrolytic S. cerevisiae expressing mutant CDT-2 and GH1-1; black triangle, $\mathbf{\Delta}$ ); D-CT2m (the phosphorolytic $S$. cerevisiae expressing mutant CDT-2 and CBP; black square, $\mathbf{\square})$. Ethanol concentration was measured in three independent experiments, and the symbols in the figure indicate average values with standard deviations. 
energy-saving effect of mutant CDT-2 was greater than that of CBP in regards to the ethanol production by D$\mathrm{CT} 2 \mathrm{~m}$. The final concentrations and yields of ethanol produced by cellobiose-fermenting S. cerevisiae expressing mutant cellodextrin transporters are summarized in Table 2.

Performance of the Phosphorolytic Cellobiose-Fermenting S. cerevisiae Expressing CDT-2 during Anaerobic SSF Contaminated by LAB

The results from micro-aerobic and anaerobic SSF of cellulose suggest that the D-CT2m showed superior ethanol production performance than those of other three cellobiose-fermenting $S$. cerevisiae strains (D-BT1m, D-CT1m and D-BT2m), regardless of SSF conditions.

Meanwhile, bacterial contamination during ethanol fermentation is a serious problem reducing ethanol yield and productivity of the biofuels production from starch, sugarcane, and cellulosic biomass [21-23]; the main bacterial contaminants are gram-positive bacteria, including LAB [20-24]. Among various LAB, L. fermentum has been found as the most dominant contaminant in bioethanol production [20,23, 24]. Since L. fermentum cannot utilize cellobiose [25], it was expected that cellobiose-fermenting yeast strain would be advantageous for mitigating the reduction of ethanol yield and productivity in SSF contaminated by L. fermentum. Consequently, SSF of cellulose contaminated by L. fermentum was performed to verify whether the D-CT2m could also show better SSF performance than those of other yeast strains, such as the traditional glucose-fermenting S. cerevisiae supplemented with extracellular $\beta$-glucosidase (D-56+188), and the hydrolytic cellobiose-fermenting $S$. cerevisiae expressing mutant CDT-1 (D-BT1m).

Fig. 3 shows the profiles of ethanol production by three yeast strains (D-BT1m, D-CT2m, and D-56+188) and lactate formation by $L$. fermentum during anaerobic SSF of Avicel PH-101 contaminated by the same number of LAB cells as the yeast cells.

In the case of LAB-contaminated SSF performed with D-56+188 strain, glucose can be primarily released from the saccharification of cellulose upon addition of Celluclast $1.5 \mathrm{~L}$ along with $\beta$-glucosidase. The D-56+188 strain might be unable to efficiently produce ethanol during the entire period of SSF because the glucose-fermenting $S$. cerevisiae might have to compete against $L$. fermentum for glucose uptake from the beginning of SSF [20,25]. Hence, the pattern of ethanol production by $\mathrm{D}-56+188$ was similar to the pattern of lactate formation by L. fermentum (like logarithmic curve shape), and the ratio for ethanol production to lactate formation remained constant throughout the entire duration of SSF (ratio for ethanol to lactate $\approx 2.0 \mathrm{~g} / \mathrm{g}$ ). The final concentrations of ethanol and lactate produced in LAB-contaminated SSF with D-56+188 were $19.9 \mathrm{~g} / \mathrm{l}$ and $10.1 \mathrm{~g} / \mathrm{l}$, respectively, as shown in Table 2.

In the case of LAB-contaminated SSF with D-BT1m strain, cellobiose can be primarily released from the saccharification of cellulose upon addition of Celluclast $1.5 \mathrm{~L}$ without $\beta$-glucosidase. This suggests that the DBT1m strain might produce ethanol efficiently at the early stage of SSF because the cellobiose-fermenting $S$. cerevisiae might not have to compete fiercely against $L$. fermentum for glucose at the beginning of SSF. However, as SSF progressed, the release of a small amount of glucose from cellulose by Celluclast 1.5L might promote the gradual activation of LAB cells growth, which might have caused several stressful and inhibitory effects on the growth and sugar metabolism of yeast cells [21,22]. Therefore, efficient production of ethanol by D-BT1m seems to have been prevented after an early period of SSF ( $32 \mathrm{~h}$ of SSF). As the results, the pattern of ethanol production
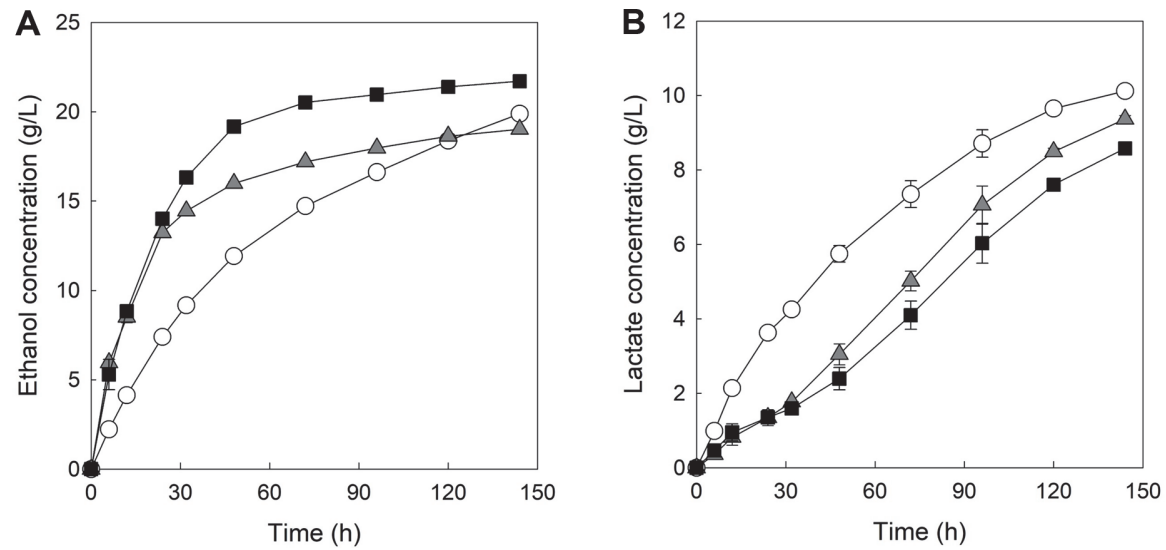

Fig. 3. Profiles of ethanol production (A) and lactate formation (B) during anaerobic SSF of $13 \%$ Avicel PH101 contaminated by lactic acid bacteria (LAB). SSF was performed by yeast cells with an OD 600 value of 30 at $30^{\circ} \mathrm{C}$ and $100 \mathrm{rpm}$. For contamination of SSF by LAB, L. fermentum cells with an OD600 value of 3 were co-inoculated with yeast cells. The yeast strains used for the SSF are as follows: D-56+188 (the parental glucose-fermenting S. cerevisiae with extracellular $\beta$-glucosidase; white circle, $\bigcirc$ ); D-BT1m (the hydrolytic S. cerevisiae expressing mutant CDT-1 and GH1-1; grey triangle, $\mathbf{A}$ ); D-CT2m (the phosphorolytic S. cerevisiae expressing mutant CDT-2 and CBP; black square, $\mathbf{\square}$ ). Celluclast 1.5L (10 FPU/g cellulose) was used for saccharification of cellulose. In SSF with D-56+188, Novozyme 188 (5.4 CBU/g cellulose) was added along with Celluclast 1.5L for degradation of cellobiose to glucose. Ethanol and lactate concentrations were measured in three independent experiments, and the symbols in the figure indicate average values with standard deviations. 
by D-BT1m exhibited hyperbolic curve shape while the pattern of lactate formation by L. fermentum was observed in a similar shape of a sigmoidal curve. In particular, the ratio for ethanol production to lactate formation of DBT1m was initially high but rapidly decreased after $32 \mathrm{~h}$ of SSF $(8.2 \mathrm{~g} / \mathrm{g}$ at $32 \mathrm{~h}, 2.5 \mathrm{~g} / \mathrm{g}$ at $96 \mathrm{~h}$ and $2.0 \mathrm{~g} / \mathrm{g}$ at $144 \mathrm{~h})$. Consequently, the hydrolytic cellobiose-fermenting S. cerevisiae strain expressing mutant CDT-1 showed an even lower production of final ethanol than that produced by the parental $S$. cerevisiae with extracellular $\beta$-glucosidase at the end of SSF $(19.0 \mathrm{~g} / \mathrm{l}$ of ethanol and $9.4 \mathrm{~g} / \mathrm{l}$ of lactate in SSF with D-BT1m) as shown in Table 2.

Remarkably, D-CT2m showed the fastest ethanol production among the strains tested during SSF contaminated by LAB. The energy-saving effects of D-CT2m based on the mutant CDT- 2 and CBP seems to have been maximized under these harsh conditions $[12,15]$, including no supply of oxygen and competition of cell growth against LAB contamination. Similar to the D-BT1m strain, D-CT2m might not have to compete fiercely against $L$. fermentum for glucose at the beginning of SSF. However, LAB cells might grow progressively over time, which might have prevented efficient production of ethanol by D-CT2m from the middle stage of SSF ( $48 \mathrm{~h}$ of SSF). As the results, similar to LAB-contaminated SSF with D-BT1m strain, ethanol production by D-CT2m and lactate formation by L. fermentum showed hyperbolic and sigmoidal curve shapes, respectively. Nonetheless, due to the energy-saving effects based on mutant CDT-2 and CBP, D-CT2m might have produced more ethanol than that produced by D-BT1m even after the middle stage of SSF. Ethanol production by D-CT2 $\mathrm{m}$ was slowed down after $48 \mathrm{~h}$ of SSF, which was $16 \mathrm{~h}$ later than time for slowdown of ethanol production observed in SSF by D-BT1m, and the ratio for ethanol production to lactate formation of D-CT2 $\mathrm{m}$ was slowly decreased compared with that of D-BT1m (ratio for ethanol to lactate in D-CT2m: $8.0 \mathrm{~g} / \mathrm{g}$ at $48 \mathrm{~h}, 3.5 \mathrm{~g} / \mathrm{g}$ at $96 \mathrm{~h}$ and $2.5 \mathrm{~g} / \mathrm{g}$ at $144 \mathrm{~h}$ ). Hence, SSF performed with D-CT2 $\mathrm{m}$ showed the highest production of ethanol and the lowest formation of lactate among all SSF experiments contaminated by LAB $(21.7 \mathrm{~g} / \mathrm{l}$ of ethanol and $8.6 \mathrm{~g} / \mathrm{l}$ of lactate in SSF with D-CT2m). Particularly, ethanol production by D-CT2m was $9 \%$ and $14 \%$ higher than those by D-56+188 and D-BT1m, respectively (Table 2).

As Fig. 33 shows that D-BT1m and D-CT2m produced similar amounts of ethanol during anaerobic SSF in the presence of $5 \mathrm{~g} / \mathrm{l}$ of lactate, compared with anaerobic SSF in the absence of lactate, it can be proposed that LAB itself (including the formation of several extracellular metabolites along with cell growth) would be the main factor inhibiting ethanol production of the cellobiose-fermenting S. cerevisiae strains rather than lactate accumulation during SSF contaminated by L. fermentum [21,22], although weak organic acid such as lactate has been known to show inhibitory effect on yeast cell growth [26]. Based on the results from LAB-contaminated SSF with the glucose- or cellobiose-fermenting yeast strains, the inhibitory effects by LAB on ethanol production could be significantly alleviated during SSF performed by D-CT2 $\mathrm{m}$ compared with SSF performed by other yeast strains. The final concentrations and yields of ethanol produced by the glucose-fermenting $S$. cerevisiae with extracellular $\beta$-glucosidase and the cellobiose-fermenting $S$. cerevisiae expressing mutant cellodextrin transporters are summarized in Table 2.

\section{Discussion}

In the previous studies, irrespective of the type of intracellular cellobiose degrading enzymes, the cellobiosefermenting yeast strains with the mutant CDT-1 (energy-dependent active cellodextrin transporter) exhibiting enhanced cellobiose transporting activity showed similar ethanol production performance during the SSF of cellulose $[8,9]$, suggesting that the cellobiose transporting activity of the cellodextrin transporter would be the key factor affecting the efficiency of the cellobiose-fermenting yeast during the SSF of cellulose.

Meanwhile, the cellobiose transporting capacity of the mutant CDT-2 (energy-independent cellodextrin facilitator) was improved to a level comparable to that of the mutant CDT-1 [15]. However, the transporting capacity of the mutant CDT-2 for cellodextrin, such as cellotriose and cellotetraose, was still lower than that of mutant CDT-1 $[12,15]$. In addition, CBP has considerably lower activity to cellodextrin than that to cellobiose, whereas GH1-1 has an as good activity to cellodextrin as that to cellobiose [11, 12]. Considering that the intermediate cellodextrin can also be released with the main product, cellobiose, from the saccharification of cellulose by cellulase enzymes during SSF, it was doubtful that the D-CT2m strain could demonstrate better ethanol production performance than those of other cellobiose-fermenting S. cerevisiae strains, such as D-BT1m and D-CT1m, during the SSF of cellulose. However, D-CT2m showed superior ethanol production performance than those of other cellobiose-fermenting S. cerevisiae strains during the SSF of cellulose.

During the micro-aerobic SSF of cellulose shown in Fig. 1, D-CT2m showed a higher ethanol production than that of D-BT2m, which was consistent with our expectations because D-BT2m had shown approximately twofold slower ethanol production due to the significant accumulation of extracellular cellodextrin during cellobiose fermentation compared with that of D-CT2m, as reported in the previous study [15]. It is interesting to note that $\mathrm{D}-\mathrm{CT} 2 \mathrm{~m}$ produced more ethanol than those produced by D-BT1m and D-CT1m, which was in contrast to our expectations because energy-saving effects of D-CT2 m based on mutant CDT-2 and CBP might be substantially suppressed under micro-aerobic conditions where the yeast cells might generate sufficient energy without tight limitation of oxygen supply. The higher ethanol production by D-CT2m than those of other yeast strains might be attributed to the enhanced $\mu_{\max }$ of D-CT2m for cellobiose, which was $12 \%, 4 \%$ and $33 \%$ higher than those of other yeast strains expressing mutant cellodextrin transporters for cellobiose, respectively $\left(\mu_{\max }\right.$ of $\mathrm{D}-\mathrm{BT} 1 \mathrm{~m}$ for cellobiose: $0.25 \mathrm{~h}^{-1} ; \mu_{\max }$ of D-CT1m for cellobiose: $0.27 \mathrm{~h}^{-1} ; \mu_{\max }$ of D-BT2m for cellobiose: $0.21 \mathrm{~h}^{-1} ; \mu_{\max }$ of DCT2m for cellobiose: $0.28 \mathrm{~h}^{-1}$ ) [8,9], as observed in Fig. S2.

During the anaerobic SSF of cellulose shown in Fig. 2, D-CT2m showed considerably higher ethanol production than that of D-BT2m, which might be because D-BT2m has fewer advantage in terms of energysaving than that of D-CT2m. It might also because D-BT2m shows significantly slower growth rate than that of D- 
CT2 $\mathrm{m}$ for cellobiose as mentioned above. Similar to the results from micro-aerobic SSF, D-CT2m showed better ethanol production than those of other yeast strains expressing mutant CDT-1, which was consistent with our expectations because energy-saving effects of D-CT2m based on mutant CDT-2 and CBP might become prominent under anaerobic conditions $[12,15]$. Considering that D-CT1m produced less ethanol than that produced by D-BT1m during anaerobic SSF, the energy-saving effects shown by D-CT2m may be primarily attributed to the mutant CDT-2 rather than CBP.

During anaerobic SSF contaminated by L. fermentum shown in Fig. 3, D-BT1m produced ethanol faster than D$56+188$ until the late stage of SSF, and D-CT2m showed the fastest ethanol production compared with other yeast strains until the end of SSF. These results suggest that the cellobiose-fermenting yeast would provide benefits, such as alleviating the reduction of ethanol yield and productivity during SSF of cellulose contaminated by LAB. In particular, D-CT2m showed the lowest lactate formation along with the highest ethanol production among the tested strains, suggesting that D-CT2m could compete most effectively against L. fermentum through the energysaving effects by mutant CDT-2 and CBP during SSF contaminated by LAB. However, L. fermentum is known as a heterofermentative lactic acid bacterium that produces $0.51 \mathrm{~g} / \mathrm{l}$ of ethanol when producing $1 \mathrm{~g} / \mathrm{l}$ of lactate [27]. This indicates that the actual concentration of ethanol produced by the cellobiose-fermenting yeast strains (when calculated by excluding the concentration of ethanol that is estimated to be produced by L. fermentum) was reduced by more than $50 \%$ during SSF contaminated by LAB compared with that observed during anaerobic SSF without LAB contamination ( $35.1 \mathrm{~g} / \mathrm{l}$ by D-BT $1 \mathrm{~m}$ and $36.3 \mathrm{~g} / \mathrm{l}$ by D-CT2m during anaerobic SSF vs. $14.2 \mathrm{~g} / \mathrm{l}$ by D$\mathrm{BT} 1 \mathrm{~m}$ and $17.3 \mathrm{~g} / \mathrm{l}$ by D-CT2 $\mathrm{m}$ during anaerobic SSF with $\mathrm{L}$. fermentum contamination). Consequently, bacterial contamination can be a serious problem that reduces ethanol yield and productivity in biofuel production [21-23]. Furthermore, utilization of the cellobiose-fermenting yeast may be limited only to certain types of SSF, such as SSF contaminated by LAB that cannot metabolize cellobiose like L. fermentum. In the case of LAB capable of metabolizing cellobiose well, such as Lactobacillus plantarum [28], the cellobiose-fermenting yeast did not effectively produce ethanol at all during SSF contaminated by L. plantarum (production of ethanol less than $5 \mathrm{~g} / \mathrm{l}$, data not shown), suggesting that other strategies should also be conceived to minimize the problems caused by LAB contamination. In addition to the utilization of the cellobiose-fermenting yeast for the SSF of cellulose, the utilization of several enzymes triggering LAB cell death by breaking down the peptidoglycan of LAB may be beneficial [29-31].

In this study, it was observed that the D-CT2m performed better during the SSF of cellulose than other yeast strains such as the cellobiose-fermenting S. cerevisiae expressing mutant CDT-1 (D-BT1m and D-CT1m). It was also observed that the D-CT2 $\mathrm{m}$ strain showed the best ethanol production and the lowest lactate formation during SSF contaminated by L. fermentum, compared with those of other strains, such as the D-BT1m and the glucosefermenting S. cerevisiae with extracellular $\beta$-glucosidase (D-56+188). Such enhanced ethanol production by the $\mathrm{D}-\mathrm{CT} 2 \mathrm{~m}$ strain may be attributed to the energetic benefits by CBP as well as mutant CDT-2. Based on the results from the current study, it can be suggested that the cellobiose-fermenting yeast employing a cellobiose metabolic pathway consuming a lower amount of energy would be useful in the SSF of cellulosic biomass for the production of biofuels and biochemicals.

\section{Acknowledgments}

This study was financially supported by Chonnam National University (Grant number: 2020-3893). This research was also supported by the National Research Foundation of Korea (NRF) grant funded by the Korea government (MSIT) (No. NRF-2019R1F1A1059516) as well as Korea Institute of Planning and Evaluation for Technology in Food, Agriculture and Forestry (IPET) and Korea Smart Farm R\&D Foundation (KosFarm) through Smart Farm Innovation Technology Development Program, funded by Ministry of Agriculture, Food and Rural Affairs (MAFRA) and MSIT, Rural Development Administration (RDA) (No. 421045-03).

\section{Conflict of Interest}

The authors have no financial conflicts of interest to declare.

\section{References}

1. Gancedo JM. 1998. Yeast carbon catabolite repression. Microbiol. Mol. Biol. Rev. 62: 334-361.

2. Jin YS, Cate JH. 2017. Metabolic engineering of yeast for lignocellulosic biofuel production. Curr. Opin. Chem. Biol. 41: 99-106.

3. Kim SR, Ha SJ, Wei N, Oh EJ, Jin YS. 2012. Simultaneous co-fermentation of mixed sugars: a promising strategy for producing cellulosic ethanol. Trends Biotechnol. 30: 274-282.

4. Liu CG, Xiao Y, Xia XX, Zhao XQ, Peng L, Srinophakun P, et al. 2019. Cellulosic ethanol production: progress, challenges and strategies for solutions. Biotechnol. Adv. 37: 491-504.

5. Subtil T, Boles E. 2012. Competition between pentoses and glucose during uptake and catabolism in recombinant Saccharomyces cerevisiae. Biotechnol. Biofuels. 5: 14.

6. Ha SJ, Galazka JM, Kim SR, Choi JH, Yang X, Seo JH, et al. 2011. Engineered Saccharomyces cerevisiae capable of simultaneous cellobiose and xylose fermentation. Proc. Natl. Acad. Sci. USA 108: 504-509.

7. Ha SJ, Wei Q, Kim SR, Galazka JM, Cate J, Jin YS. 2011. Cofermentation of cellobiose and galactose by an engineered Saccharomyces cerevisiae strain. Appl. Environ. Microbiol. 77: 5822-5825.

8. Lee WH, Jin YS. 2017. Improved ethanol production by engineered Saccharomyces cerevisiae expressing a mutated cellobiose transporter during simultaneous saccharification and fermentation. J. Biotechnol. 245: 1-8.

9. Lee WH, Jin YS. 2017. Evaluation of ethanol production activity by engineered Saccharomyces cerevisiae fermenting cellobiose through the phosphorolytic pathway in simultaneous saccharification and fermentation of cellulose. J. Microbiol. Biotechnol. 27: 1649-1656. 
10. Lee WH, Nan H, Kim HJ, Jin YS. 2013. Simultaneous saccharification and fermentation by engineered Saccharomyces cerevisiae without supplementing extracellular $\beta$-glucosidase. J. Biotechnol. 167: 316-322.

11. Galazka JM, Tian C, Beeson WT, Martinez B, Glass NL, Cate JH. 2010. Cellodextrin transport in yeast for improved biofuel production. Science 330: 84-86.

12. Ha SJ, Galazka JM, Oh EJ, Kordić V, Kim H, Jin YS, et al. 2013. Energetic benefits and rapid cellobiose fermentation by Saccharomyces cerevisiae expressing cellobiose phosphorylase and mutant cellodextrin transporters. Metab. Eng. 15: 134-143.

13. Parisutham V, Chandran SP, Mukhopadhyay A, Lee SK, Keasling JD. 2017. Intracellular cellobiose metabolism and its applications in lignocellulose-based biorefineries. Bioresour. Technol. 239: 496-506.

14. Kim H, Lee WH, Galazka JM, Cate JH, Jin YS. 2014. Analysis of cellodextrin transporters from Neurospora crassa in Saccharomyces cerevisiae for cellobiose fermentation. Appl. Microbiol. Biotechnol. 98: 1087-1094.

15. Kim H, Oh EJ, Lane ST, Lee WH, Cate JH, Jin YS. 2018. Enhanced cellobiose fermentation by engineered Saccharomyces cerevisiae expressing a mutant cellodextrin facilitator and cellobiose phosphorylase. J. Biotechnol. 275: 53-59.

16. Alexander JK. 1961. Characteristics of cellobiose phosphorylase. J. Bacteriol. 81: 903-910.

17. Tewari YB, Goldberg RN. 1989. Thermodynamics of hydrolysis of disaccharides: cellobiose, gentiobiose, isomaltose, and maltose. J. Biol. Chem. 264: 3966-3971.

18. Oh EJ, Kwak S, Kim H, Jin YS. 2017. Transporter engineering for cellobiose fermentation under lower pH conditions by engineered Saccharomyces cerevisiae. Bioresour. Technol. 245: 1469-1475.

19. Hosaka K, Nikawa JI, Kodaki T, Yamashita S. 1992. A dominant mutation that alters the regulation of INO1 expression in Saccharomyces cerevisiae. J. Biochem. 111:352-358.

20. Bischoff KM, Liu S, Leathers TD, Worthington RE, Rich JO. 2009. Modeling bacterial contamination of fuel ethanol fermentation. Biotechnol. Bioeng. 103: 117-122.

21. Beckner M, Ivey ML, Phister TG. 2011. Microbial contamination of fuel ethanol fermentations. Lett. Appl. Microbiol. 53: 387-394.

22. Brexó RP, Sant'Ana AS. 2017. Impact and significance of microbial contamination during fermentation for bioethanol production. Renew. Sust. Energ. Rev. 73: 423-434.

23. Lucena BT, dos Santos BM, Moreira JL, Moreira APB, Nunes AC, Azevedo V, et al. 2010. Diversity of lactic acid bacteria of the bioethanol process. BMC Microbiol. 10: 298.

24. Liu M, Bischoff KM, Gill JJ, Mire-Criscione MD, Berry JD, Young R, et al. 2015. Bacteriophage application restores ethanol fermentation characteristics disrupted by Lactobacillus fermentum. Biotechnol. Biofuels 8: 132.

25. Verce M, De Vuyst L, Weckx S. 2020. Comparative genomics of Lactobacillus fermentum suggests a free-living lifestyle of this lactic acid bacterial species. Food. Microbiol. 89: 103448.

26. Piper PW. 2011. Resistance of yeasts to weak organic acid food preservatives. Adv. Appl. Microbiol. 77: 97-113.

27. Lahtinen S, Ouwehand AC, Salminen S, von Wright A. 2011. Lactic acid bacteria: microbiological and functional aspects. Crc Press.

28. Ucar RA, Pérez-Díaz IM, Dean LL. 2020. Gentiobiose and cellobiose content in fresh and fermenting cucumbers and utilization of such disaccharides by lactic acid bacteria in fermented cucumber juice medium. Food Sci. Nutr. 8: 5798-5810.

29. Khatibi PA, Roach DR, Donovan DM, Hughes SR, Bischoff KM. 2014. Saccharomyces cerevisiae expressing bacteriophage endolysins reduce Lactobacillus contamination during fermentation. Biotechnol. Biofuels 7: 104.

30. Kim JS, Daum MA, Jin YS, Miller MJ. 2018. Yeast derived LysA2 can control bacterial contamination in ethanol fermentation. Viruses 10: 281 .

31. Roach DR, Khatibi PA, Bischoff KM, Hughes SR, Donovan DM. 2013. Bacteriophage-encoded lytic enzymes control growth of contaminating Lactobacillus found in fuel ethanol fermentations. Biotechnol. Biofuels 6: 20. 